\title{
Research on Cross-Cultural Marketing of Acupuncture and Moxibustion Based on the Belt and Road Initiative
}

\author{
Zeng Zhi \\ Nanjing University of Chinese Medicine, College of Health Economics and Management, Nanjing, China \\ Email address: \\ zengzhinju@qq.com \\ To cite this article: \\ Zeng Zhi. Research on Cross-Cultural Marketing of Acupuncture and Moxibustion Based on the Belt and Road Initiative. Humanities and \\ Social Sciences. Vol. 7, No. 2, 2019, pp. 67-70. doi: 10.11648/j.hss.20190702.13
}

Received: March 19, 2019; Accepted: June 11, 2019; Published: June 15, 2019

\begin{abstract}
The promotion and dissemination of traditional Chinese medicine culture is an important component of the national science and technology link along the Belt and Road as well as the basis for building a community of healthy destinies. In recent years, there has been a worldwide boom in acupuncture academic research, acupuncture cross-cultural marketing has developed rapidly. Understanding and grasping the development status of acupuncture and moxibustion in foreign countries and its future development trends are of great significance to how acupuncture and moxibustion go further into the world and also greatly promote and represent the status and impact of cross-cultural communication of "Chinese elements".
\end{abstract}

Keywords: Chinese Elements, The Belt and Road, Acupuncture, Cross-Culture

\section{Introduction}

Chinese medicine is a treasure of ancient Chinese science, is a key to open the treasure house of Chinese civilization, and is a forerunner of the great rejuvenation of Chinese civilization. With the development of the time, the exchanges between countries are now more and more frequent, and the cross-cultural industries in various countries are flourishing in our country. Similarly, in order to carry forward our traditional culture and integrate it with the economy, it is necessary to understand the cross-cultural marketing of some industries that contain "elements of China." "Acupuncture and Moxibustion Medicine" has been spread to foreign countries for thousands of years. With the growing power of our country and the improvement of our right to speak, "Chinese elements" have also gradually drawn the attention of all parties. In particular, acupuncture and moxibustion are of great significance in safeguarding human health and preventing and treating diseases Achievements and impacts have drawn more and more attention and attention from the international community. In recent years, the research boom in acupuncture and moxibustion has been set off in the world. The cross-cultural marketing research on acupuncture and moxibustion has developed rapidly.

\section{Acupuncture Development History and Status}

Acupuncture originated in China, which has a long history. Acupuncture originated in the legendary era of the Three Emperors and Five Emperors, Fuxi invented the acupuncture, "Hundreds of herbs and nine needle system" [1]. According to "Shanhai Jing" ("Shan Hai Jing" is an important ancient book of the pre-Qin period in China. It covers ancient astronomy, geography, birds and beasts, folk customs, myths, etc. It is the earliest encyclopedia of ancient culture.), "Nei Jing"("Nei Jing" is also known as the "Yellow Emperor's Internal Classic". It was written in the Warring States to the Qin and Han Dynasties. It is the earliest extant TCM theory book in China. It is a summary of the medical experience and theoretical knowledge before the Spring and Autumn Period.) on the "stone" pierce the tumor record, "Mencius" in the "seven years of disease, seeking three years of $\mathrm{Ai}^{\prime \prime}$ argument and archaeological evidence of historical relics Acupuncture therapy can be traced back to the Stone Age. According to records, when people got the pain and felt discomfort, they did not consciously use their hands or even sharp stone massage, hammering, the earliest needle - stone also came into being. The moxibustion rule comes after the discovery and use of fire. 
In the process of using fire, people at that time found that the pain in the body parts was relieved by the burning of the fire and gradually learned to use partial barks or skins to wrap the hot clods. And then gradually developed to ignite the branches or wormwood to treat pain, future generations after long-term exploration to explore, easy to burn but can warm the meridians of Artemisia gradually became the main material moxibustion. In other words, the "needle" and "moxibustion" from the beginning belong to different therapies. Later, "thorn and thorn" gradually developed into acupuncture, "hot and iron" gradually developed into moxibustion, which is the predecessor of acupuncture therapy [2].

Acupuncture therapy is gradually formed in the long history, and acupuncture-related academic thinking are also gradually accumulated with the accumulation of clinical medicine experience. From "Su Wen" to "Lingshu"( "Lingshu" and "Su Wen" are two important theoretical medical books in Chinese traditional culture. They are collectively referred to as the "Yellow Emperor's Canon." Su Wen mainly talks about yin and yang five elements, Tibetan elephant theory, etiology and pathogenesis, health and disease prevention, etc. Lingshu mainly talks about acupuncture, acupoints and viscera.), from "acupuncture and meridian A" to "acupuncture and moxibustion of bronze acupuncture points", from "the exegesis of the fourteen" to "the acupuncture Dacheng" medical people spend countless effort. The status of the medical community had nothing comparable to that until the Ming and Qing Dynasties acupuncture and moxibustion therapy gradually decline from prosperity [3]. In $1742 \mathrm{Wu}$ Qian et al. Wrote the "Medical Examination of the Golden Mirror," which inherited not only the acupuncture points of ancient sages, but also flourished. However, in the late Qing Dynasty, the rulers ordered the hospital to ban the use of acupuncture for the absurd reasons of "acupuncture fire moxibustion and non-compliance with non-imperialism." After the Opium War, acupuncture was even more devastated. When the government ordered the repeal of Chinese medicine during the Republican period, acupuncture was even more devastating. [4]

Fortunately, since the founding of the People's Republic of China, the Chinese government has attached great importance to inheriting and carrying forward the precious medical heritage of Chinese traditional culture and has adopted a series of policies to protect the cause of Chinese medicine. In this era, acupuncture and moxibustion medicine has been popularized and improved.

\section{The Role of Acupuncture}

Acupuncture is an ancient and mysterious science. Our ancestors invented the acupuncture and acupuncture needles. The spread of acupuncture and moxibustion has lasted for thousands of years and acupuncture and moxibustion has the highest status in Chinese medicine system. It is the most representative one.

\subsection{Reconcile the Yin and Yang}

Acupuncture and Moxibustion reconcile Yin and Yang refers to the meridians, acupoints and acupuncture techniques to eliminate the imbalance of yin and yang. The pathology of the disease is extremely complex, but generally it can be summarized as an imbalance of yin and yang. "Yin yang is yang disease, yang yang is yin yang." When Yin and Yang and other factors lead to the human body is not adjusted, loss of relative balance, organs and meridians function will abnormal activity, thereby inducing the disease. The use of acupuncture to adjust the balance of yin and yang, so as to achieve the purpose of the patient recovered.

\subsection{Dredge the Meridians}

Dredging the meridians and collaterals can make the meridians and collaterals unimpeded to play their normal physiological function, which is the most basic and direct therapeutic effect of acupuncture. The meridian "Inside the house is dirty, outside the collaterals in the Limb section" Operation Qi and Blood is one of the basic physiological functions of Meridian, so when the meridians and collaterals stasis, qi and blood run not smooth, then the human body naturally unwell, appear disease phenomenon. Acupuncture dredging meridians are mainly based on the operation of the meridians to choose the corresponding acupoints and acupuncture methods and trigonous needle point bleeding,-tapping, cupping, etc. so that the meridians dredge, QI and blood running smoothly, to achieve the purpose of patient's illness.

\subsection{Fuzheng Quxie}

Acupuncture righting Quxie role is to help the body righteousness and get rid of evil. Acupuncture medical treatment believes that the emergence, development and rehabilitation of the disease are actually the process of fighting between the true and the evil. The so-called evil canada goose jakke, regular disease has been eased, is beyond the evil of the disease continued to increase, therefore, righteousness is the basic guarantee of disease relief is the role of acupuncture treatment of disease process. Fighting evil in the clinical remedy is achieved through the tonic.

\section{Cross-Cultural Marketing of Acupuncture and Its Influencing Factors}

\subsection{Acupuncture Cross-Cultural Marketing Status}

As early as the 6th century $\mathrm{AD}$, acupuncture and moxibustion in China began to spread abroad. At present, more than 120 countries and regions have used acupuncture for the treatment of their own people. Many countries have set up acupuncture academic groups, acupuncture educational institutions and research institutions to study and promote acupuncture. According to reports, acupuncture 
effective treatment of 307 kinds of diseases, of which there are significantly more than 100 kinds of effects. In 1980, the United Nations World Health Organization put forward 43 kinds of recommended acupuncture treatment of adaptation disorders. In 1987, the World Federation of Acupuncture and Moxibustion was formally established in Beijing, and acupuncture and moxibustion as the world's prevalent medical science was established in the world [7].

It is not necessary to elaborate on the grievances in China and Japan. In $562 \mathrm{AD}, \mathrm{Wu}$ Renzhi from the Southern and Northern Dynasties carried "Mingtangtu" and other 160 volumes of medical books to Japan, which is considered as the beginning of Japanese acupuncture medicine. . France is one of the earliest countries to use acupuncture in traditional Chinese medicine in Europe. Acupuncture with clinical significance began in the 1930s. So far, the number of acupuncture doctors preparing records has been quite large, and the number of non-medical practitioners even more, Which I have to say is a great success of the development of acupuncture [9]. Previously Korea and China compete for acupuncture "intangible heritage" right is despicable, but also illustrates the side effects of acupuncture in Korea [10]. The National Institutes of Health published the "NIH Statement of Uniformity in Acupuncture and Moxibustion," which also states that acupuncture has a significant effect on multiple conditions [11].

Of course, acupuncture is not a very smooth cross-cultural marketing in every country. In general, the scope of application of acupuncture in various countries abroad is relatively large. As acupuncture treatment and use of a wide range of methods, such as cupping, moxibustion, Ginger moxibustion, body acupuncture, point pressure, etc., each has its own advantages and disadvantages, so not every one is so popular. Such as moxibustion moxibustion because of its relatively large number of patients are not accepted. And it must be admitted that acupuncture medicine in many countries academic status is much lower than the Western medicine, not be taken seriously. In some countries acupuncture can only be practiced by Western medicine practitioners. Even acupuncturists in some countries can not enter the hospital for official posts but only work in clinics. Their social status and economic income are far lower than that of western medicine practitioners [12].

\subsection{Acupuncture Cross-Cultural Marketing Factors}

Acupuncture cross-cultural marketing there are many factors, the first is that acupuncture in the foreign medical profession is not high status, but many countries acupuncture as Western medicine "supplement" fills. If not all kinds of treatment are not satisfied with the efficacy, the general foreign patients seldom take the initiative to seek acupuncture treatment. This also led to foreign people on the efficacy of acupuncture treatment is not well understood, cognitive rate underground, so a large number of foreign acupuncture professionals are buried, under the vicious circle of Western medicine rose, acupuncture potential weak, other people can not see the acupuncture significantly Efficacy results will Acupuncture is only positioned as Western medicine "supplementary medicine", thus more fundamentally limited the development of acupuncture. Second, it is clear that acupuncture education is not fully recognized abroad. Although many countries have set up acupuncture-specialized universities and research institutes, it is a pity that many acupuncture graduates, even though they eventually qualify as national acupuncturists because of a lack of clinical skills training and internship training at school, After graduation, it is difficult to obtain further education and further self-improvement opportunities. Therefore, acupuncture graduates who truly become an acupuncturist abroad are few.

Then there is the language and culture barrier already mentioned above. Due to the different language and cultural backgrounds, ordinary people in other countries want to know about acupuncture is very difficult. As we all know, our acupuncture inherited from ancient times all the way, the earliest records come from ancient books. Obscure language is obscure, that is, our people and non-people can understand, and then consider the inheritance of ancient copyrighted omissions and other omissions, acupuncture and learning is even more difficult. What's more, the medical class of ancient books, though not as literary classics as the general "who also" and ultimately, some biting, look tired dear. Moreover, all generations of people in different generations of China, because of the inconvenience of information exchange, were not unified with the names of medicinal materials and acupuncture points. Even before the unification, they did not even have a unified text, which caused great impediments to the spread of acupuncture and moxibustion medicine. Nowadays, although people of insight bring our acupuncture and moxibustion medicine abroad and translate them, acupuncture and moxibustion terms are still abstract and obscure in general, making people find it hard to understand.

For the spread of acupuncture, national and local government support is essential. Without the support of the government, any new things will be struggling early on. As in the early 1970s, acupuncture in China was "overwhelming" in the United States. In the absence of legal protection, many states in the United States targeted acupuncture as "illegal practice" in the early 1970s. Li Chuanzhen, one of the earliest promoters of the development of acupuncture in California, was hit by a case of "unlicensed practice." Although Li Chuan-shing was later called "the mother of acupuncture" in the California acupuncture community, it can not change the case. Cultural marketing caused a great blow, and even became a stain that can not be washed [13].

Acupuncture is one of the traditional Chinese medicine systems. Nowadays, Western medicine is prevalent. Even Chinese people who choose the most effective first-choice Western medicine soon after their illness. After all, our country is now in a fast-paced society, where "time is money" and "thinking of every minute and second" are implemented in our hearts. Therefore, although many Chinese medicine methods have wonders repeatedly, they have been abandoned for a long time. Fortunately, acupuncture and effective 
treatment is not slow, in many parts of our country there are many specialized acupuncture clinic, acupuncturist in the hospital also sits. Unfortunately, many patients or physicians still choose Western medicine. At least the author has to go directly to the hospital in the past few years, doctors will directly select the Western medical treatment, but more unfortunately, acupuncture clinic has not been fully popularized, although the status has improved recently, but acupuncture clinic is not easily find many well-known acupuncturist Acupuncture clinics have to suffer from people's word of mouth.

In addition, the development of the times, acupuncture is certainly our traditional culture, but also with the progress of the times together, and some of the modern high-tech combination, so that patients can improve the acupuncture therapy sense of security, identity. Acupuncture is no longer considered psychological effects but no actual effect. When they are willing to deliver their trust in acupuncture, more and more people are willing to take acupuncture medicine to safeguard their health and save their lives. At this point, acupuncture and medicine cross-cultural marketing can stride forward.

\section{Conclusion}

The Belt and Road initiative is a historical necessity for Reform and Opening-up and lead economic globalization in china. And it is a cultural awareness of China's active participation in the world development process. In the specific implementation of the Belt and Road initiative, Chinese medicine has become an ideal object to study Chinese culture and an ideal carrier for Chinese culture to "go out". Under the impetus of the Belt and Road, it is necessary to understand the cross-culture marketing of some industries containing "Chinese elements" in order to carry forward our traditional culture and combine it with the economy. Traditional Chinese medicine is the treasure of ancient Chinese science and the key to open the treasure house of Chinese civilization. As the most characteristic medical technology of traditional Chinese medicine, the achievements and effects of acupuncture and moxibustion in maintaining human health and preventing diseases have been paid more and more attention by the international community. Acupuncture and moxibustion have become an important carrier of cross-cultural marketing of traditional Chinese medicine.

In the process of cross-cultural marketing of acupuncture and moxibustion, we should first further establish the status of acupuncture and moxibustion in the international medical community, and let more countries accept the way of acupuncture and moxibustion treatment of diseases. Secondly, we should strengthen medical education, enhance people's understanding of acupuncture and moxibustion in the world through knowledge sharing and dissemination, to accept acupuncture and moxibustion treatment. Thirdly, we need to understand the culture of other countries and eliminate cultural distance, especially to win the recognition of relevant government departments. With the deepening of the Belt and Road initiative in practice, acupuncture as a symbol of Chinese elements will achieve gratifying achievements in cross cultural marketing.

\section{Acknowledgements}

This research was funded by Jiangsu Philosophy and Social Science Foundation (Project Number: 16GLB012).

\section{References}

[1] Tang Lewei, Chen Liang et al. Development and evolution of acupuncture rules and theoretical value [J] Journal of Traditional Chinese Medicine, 2014, 55 (9): 728-731.

[2] Guo Tai Pin, Ren Yulan et al. Inquiry acupuncture and its transformation of the use of [J] Journal of Traditional Chinese Medicine, 2014, 55 (12): 999-1002.

[3] Yue Feng first. Chinese rule "sick person" Western treatment of "human disease" [N] Life Times, 2010-03-03 (3).

[4] Chen Kaijia, Deng Tietao. Guoyi master Deng Tietao traditional Chinese medicine education thinking [J] Journal of Traditional Chinese Medicine, 2012, 30 (8): 1715-1716.

[5] Yu Yanping. Acupuncture and moxibustion on the effect of [J] Shanxi Traditional Chinese Medicine, 2012, 33 (3): 328-330.

[6] Yang Huayuan, Ma Yini. Current state of the art of acupuncture treatment equipment $[\mathrm{J}]$. Chinese Medical Equipment, 2011, 26 (4): 46-49.

[7] Wang Jing. The first release of ISO Chinese medicine international standards [J] Journal of Traditional Chinese Medicine, 2014, 22 (2): 249.

[8] Li Ruiyao, Li Hongyuan, Li Junde. Overview of the development of Chinese medicine in Japan [J] World Journal of Integrated Traditional and Western Medicine, 2016, 11 (3).

[9] Wang Liming, Liu Xiaofei. A cross-cultural perspective on the development of acupuncture in France $[\mathrm{J}]$ French Studies, 2013, (2): 94-99.

[10] Liu Guowei. Analysis of traditional Chinese medicine cross-cultural transmission [J] Chinese Medicine, 2011, 26 (5): 1047-1050.

[11] In Tianyi. Chinese medicine and acupuncture in the United States of communication and development [J], 2011, 27 (11): 998-1001.

[12] Yin Shuai, Wu Nan, etc. From the perspective of cultural transmission on the issue of international development of acupuncture [J] World Chinese Medicine, 2015, 4 (5): 87-88.

[13] Jiang Jibiao. Overseas Chinese medicine center development strategy $[\mathrm{J}]$. World Integrative Medicine, 2016, 11 (4): $567-569,575$. 